\title{
Effect of 7,8-Dihydroneopterin, Biopterin and Isoxanthopterin on Cholesterol and Phospholipid Content and Phospholipid Biosynthesis in Vitro
}

\author{
Vera Rudzite, Edite Jurika, Gabriele Baier-Bitterlich ${ }^{1}$, Helmut Wachter $^{1}$, Dietmar Fuchs ${ }^{1 \S}$
}

Latvia Institute of Cardiology, Riga, Latvia and ${ }^{1}$ Institute of Medical Chemistry and Biochemistry, University of Innsbruck, Innsbruck, Austria

(Received March 2, 1996)

\begin{abstract}
Summary
Incorporation of fatty acids into phospholipids has been investigated using samples of rat liver tissue homogenate (Krebs-Ringer-phosphate buffer, $\mathrm{pH}=7.4$, containing $0.3 \%$ albumin, fatty acid mixture and glycerol). The addition of 7,8-dihydroneopterin and biopterin (5 and $30 \mathrm{pmol} / \mathrm{g}$ wet weight) to incubation medium induced a decrease of palmitic (7,8-dihydroneopterin) and stearic acid (7,8-dihydroneopterin, biopterin) and an increase of linolenic (7,8-dihydroneopterin) and arachidonic acid (7,8dihydroneopterin, biopterin) incorporation into phospholipids. Cholesterol content decreased but phospholipid content did not change in samples containing 7,8-dihydroneo-pterin or biopterin. No changes of fatty acid incorporation into phospholipids as well as of the content of cholesterol and phospholipids were observed in samples after addition of isoxanthopterin ( 5 and $30 \mathrm{pmol} / \mathrm{g}$ wet weight) to incubation medium for phospholipid biosynthesis in vitro. Despite the fact that 7,8-dihydroneopterin and biopterin have different oxidation states and some similar effects on phospholipid biosynthesis and cholesterol content have been observed in our study, we suggest that the oxidation state of pteridines is more important than the pterin-(6)-side chain in the deviations of fatty acid incorporation into phopholipids as well as in the regulation of cholesterol content in samples.
\end{abstract}

Key Words : 7,8-dihydroneopterin, Biopterin, Isoxanthopterin, Fatty acids, Phospholipids, Cholesterol

\section{Introduction}

The results of our previous observations (1, 2, 3 ) showed that kynurenine, neopterin, sepiapterin, 7, 8-dihydroneopterin and 5, 6, 7, 8-tetrahydrobiopterin have different effects on phospholipid biosynthesis as well as cholesterol con-

§ Address for correspondence: Dr. Dietmar Fuchs, Institute of Medical Chemistry and Biochemisty University of Innsbruck, Friz Pregl Strasse 3, A-6020 Innsbruck, Austria tent. L-kynurenine and D-neopterin promoted the incorporation of saturated (kynurenine, neopterin) and mono-unsaturated (kynurenine) fatty acids into phospholipids and elevated cholesterol content in samples while sepiapterin, 7, 8-dihydrobiopterin and 5,6,7,8-tetrahydrobiopterin had the opposite effect. They promoted incorporation of polyunsaturated fatty acids, especially of arachidonic acid, into phospholipids and decreased the cholesterol content in samples. For providing the evidence whether the configuration 
of pterin-(6)-side chain or the oxidation state of pteridines is more important for the effects seen by us further studies were needed. The purpose of this study was to examine the effects of 7,8 dihydroneopterin, biopterin and isoxanthopterin on phospholipid biosynthesis in vitro as well as on cholesterol and phospholipids content.

\section{Methods}

Homogenized liver tissue of 12 male Wistar rats in Krebs-Ringer-phosphate buffer $(\mathrm{pH}=7.4)$ containing $0.3 \%$ albumin were used for these experiments (1-4). Samples were incubated two times at $37^{\circ} \mathrm{C}$ for 4 hours. To investigate the effect of different pteridines, 7,8-dihydroneopterin ( 5 and $30 \mathrm{pmol} / \mathrm{g}$ wet weight), biopterin ( 5 and $30 \mathrm{pmol} / \mathrm{g}$ wet weight) and isoxanthopterin (5 and $30 \mathrm{pmol} / \mathrm{g}$ wet weight) were added to experimental test tubes before the first incubation. All pteridine derivatives were obtained from Schircks Laboratories, Jona, Switzerland.

All the metabolites were diluted with bidistilled water. The same volume of bidistilled water $(0.1$ $\mathrm{ml}$ ) without addition of metabolites was added to the control test tubes. After the incubation at $37^{\circ} \mathrm{C}$ for 4 hours $60 \mu \mathrm{l}$ of $20 \%$ fatty acid solution in chloroform (stearic, palmitic, linoleic, oleic and arachidonic acid in equivalent amounts; SIGMA, Munich, Germany) and $40 \mu \mathrm{l}$ of glycerol were added to all test tubes. Then the test tubes were again incubated at $37^{\circ} \mathrm{C}$ for 4 hours. After this second incubation, $75 \mathrm{ml}$ of chloroform/ methanol mixture $(2: 1)$ was added to each sample. The samples were mixed and left for $40 \mathrm{~min}$ at room temperature. Then the chloroform/ methanol extract was filtered and the filtrate was carefully treated according to the method of Folch et al. (5). The whole cholesterol concentration was determined by the method of Engelhard and Smirnova (6), the amount of phospholipids-by the method of Urbach-Raabe (7). The method of Kates (8) was used to prepare the phospholipid samples for fatty acid examination by gas chromatography. Helium was used as a carrier gas and the sorbent was chromosorb covered with DEAG. Separated fatty acid methylesters were monitored at $210^{\circ} \mathrm{C}$, using flame ionization detection and an adequate flow rate of helium. The separation was controlled by internal methylester standard substances (SERVA, Buchs, Switz erland).

Statistical analyses were done using Student's ttest. P-values below 0.05 were considered to in-

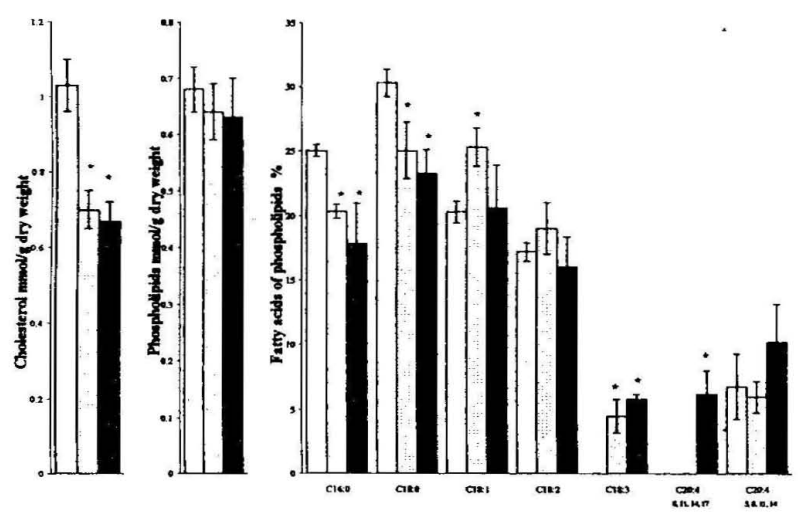

Figure 1. Concentrations (mean \pm S.D.) of cholesterol and phospholipids, as well as fatty acid content (in percent: mean \pm S.D.) of phospholipids in controls (open bars) and in samples with $5 \mathrm{pmol} / \mathrm{g}$ wet weight (dotted bars) or with $30 \mathrm{pmol} / \mathrm{g}$ wet weight (hatched bars) 7,8 dihydroneopterin. Samples of rat liver tissue homogenates were used to test for phospholipid biosynthesis in vitro. Incubation medium: Krebs-Ringer-phosphate buffer $(\mathrm{pH}=7.4)$, fatty acid mixture and glycerol. Samples were incubated twice for $4 \mathrm{~h}$ at $37^{\prime \prime} \mathrm{C}$. * The changes are statistically significant $(p<0.002)$.

dicate significant differences (9).

\section{Results}

Fig. I shows the changes of phospholipid biosynthesis after addition of 7,8 -dihydroneopterin (5 and $30 \mathrm{pmol} / \mathrm{g}$ wet weight) to experimental samples. We have observed a decrease of palmitic $(\mathrm{p}<0$. $001)$ and stearic acid $(\mathrm{p}<0.002)$ and an increase of linolenic acid $(\mathrm{p}<0.001)$ incorporation into phospholipids of incubated experimental samples. We have also observed the $8,11,14,17$-arachidonic acid in incubated samples with $30 \mathrm{pmol} / \mathrm{g}$ wet weight 7,8 -dihydroneopterin, while control samples contained only $5,8,11,14$-arachidonic acid. Addition of 7,8-dihydroneopterin to incubation medium did not change the phospholipid content in samples, while the cholesterol content significantly decreased $(\mathrm{p}<0.001)$.

Fig. 2 points out the deviation of phospholipid biosynthesis after the addition of biopterin 15 and $30 \mathrm{pmol} / \mathrm{g}$ wet weight to incubation medium. We have obsened a decrease of stearic acid $(\mathrm{p}<$ $0.001)$ and an increase of arachidonic acid ( $\mathrm{p}<$ 0.001 ) incorporation into phospholipids. Chromatograms of experimental samples showed the appearance of $5,8,11,14$-arachidonic acid as well as $8,11,14,17$-arachidonic acid in high concentration, while control samples contained only 5 , $8,11,14$-arachidonic acid. The cholesterol con- 


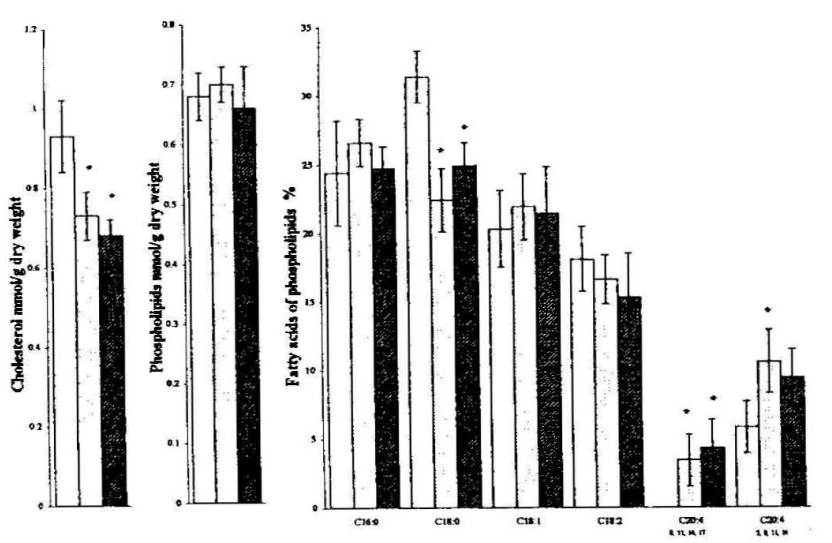

Figure 2. Concentrations (mean \pm S.D.) of cholesterol and phospholipids, as well as fatty acid content (in percent: mean \pm S.D.) of phospholipids in controls (open bars) and in samples with $5 \mathrm{pmol} / \mathrm{g}$ wet weight (dotted bars) or with $30 \mathrm{pmol} / \mathrm{g}$ wet weight (hatched bars) biopterin. Samples of rat liver tissue homogenates were used to test for phospholipid biosynthesis in vitro. Incubation medium: Krebs-Ringer-phosphate buffer ( $\mathrm{pH}=7$. 4 ), fatty acid mixture and glycerol. Samples were incubated twice for $4 \mathrm{~h}$ at $37^{\circ} \mathrm{C}$. * The changes are statistically significant $(\mathrm{p}<0.001)$.

tent in samples containing biopterin decreased $(\mathrm{p}$ $<0.001$ ) but the amount of phospholipids remained unchanged.

Fig. 3 reflects the influence of isoxanthopterin ( 5 and $30 \mathrm{pmol} / \mathrm{g}$ wet weight) on phospholipid biosynthesis in vitro. The addition of isoxanthopterin to samples for phospholipid biosynthesis neither altered incorporation of fatty acids into phospholipids nor the content of cholesterol and phospholipids.

\section{Discussion}

The results of our earlier observations (1) showed that the addition of D-erythro-neopterin ( 5 and $30 \mathrm{pmol} / \mathrm{g}$ wet weight) to incubation medium for phospholipid biosynthesis in vitro was followed by an increase of palmitic acid and a decrease of oleic and linoleic acid incorporation into phospholipids as well as by appearance of linolenic acid in phospholipids of samples. These changes were accompanied by an increase of cholesterol content in samples. The results of this study point out that the addition of 7,8 -dihydroneopterin, the reduced form of neopterin, is followed by the opposite effect. It induces a decrease of saturated fatty acids (palmitic and stearic acid) incorporation into phospholipids, while the incorporation of oleic and linoleic acid into phos-

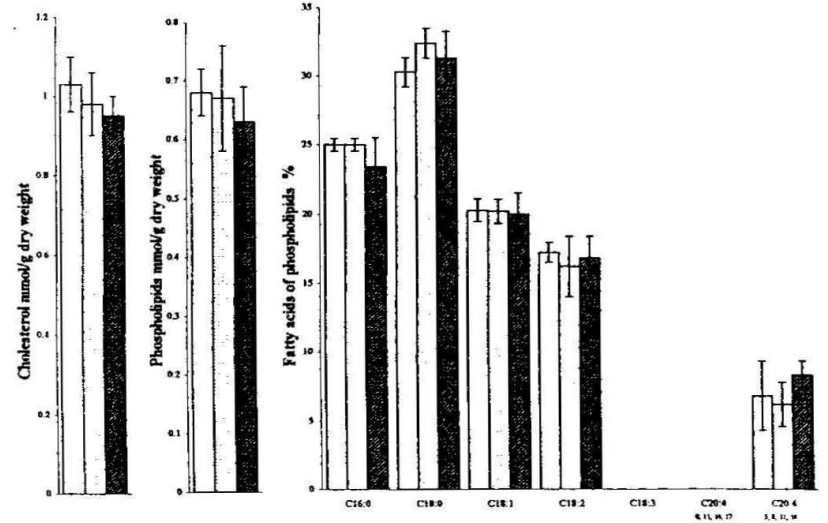

Figure 3. Concentrations (mean \pm S.D.) of cholesterol and phospholipids, as well as fatty acid content (in percent: mean \pm S.D.) of phospholipids in controls (open bars) and in samples with $5 \mathrm{pmol} / \mathrm{g}$ wet weight (dotted bars) or with $30 \mathrm{pmol} / \mathrm{g}$ wet weight (hatched bars) isoxanthopterin. Samples of rat liver tissue homogenates were used to test for phospholipid biosynthesis in vitro. Incubation medium: Krebs-Ringer-phosphate buffer ( $\mathrm{pH}$ =7.4), fatty acid mixture and glycerol. Samples were incubated twice for $4 \mathrm{~h}$ at $37^{\circ} \mathrm{C}$.

pholipids is not altered. The appearance of linolenic acid in phospholipids was the same as in cases with neopterin (1). Moreover, we have observed the appearance of $8,11,14,17$-arachidonic acid in samples incubated with 7,8-dihydroneopterin. This type of arachidonic acid develops from linolenic acid, while 5,8,11, 14-arachidonic acid derives from linoleic acid (10). The changes in phospholipid biosynthesis induced by 7,8 -dihydroneopterin are followed by a decrease of cholesterol content in samples.

The results obtained after addition of 7, 8-dihydroneopterin to the incubation medium for phospholipid biosynthesis in vitro are similar to those observed in our earlier investigations after the ad dition of reduced forms of biopterin (sepiapterin, 7,8-dihydrobiopterin and 5, 6, 7,8-tetrahydrobiopterin) to the same assay system (3). Recently it was found that pterin derivatives are $\mathrm{ca}^{-}$ pable of interfering with reactive oxygen compounds, e.g. neopterin was found to enhance hydrogen peroxide induced chemiluminescence in the presence of chelated iron at neutral or slightly alkaline $\mathrm{pH}$, whereas 7,8 -dihydroneopterin scavenges this reaction (11, 12). Further studies revealed that pterin derivatives in their oxidized form like neopterin, monapterin or biopterin are generally potent enhancers whereas 7,8-dihydro forms generally act as scavengers (13). The results obtained with our tests system share some similarities with the findings made with chemi- 
luminescence mcasurements: pterin species show a different behaviour which is obviously related to the oxidation status of carbons 7 and 8: e.g., oxidized forms seem to promote incorporation of saturated fatty acids into phospholipids and increase the cholesterol content whereas 7,8-dihydroforms promote incorporation of polyunsaturated fatty acids into phospholipids and decrease the cholesterol content. With the exception of results obtained with biopterin, our data suggest that the capacity of pteridine derivatives to interfer with the biosynthesis of phospholipids is somewhat related to their influence on reactive oxygen compounds.

When comparing the results of the present study with the data observed earlier using neopterin in the same assay system (1), we saw an inverse relationship between the effects induced by biopterin in relation to neopterin: biopterin supported the incorporation of polyunsaturated fatty acids, especially arachidonic acid, into phospholipids and decreased cholesterol formation. Interestingly both, biopterin and neopterin, have a very similar molecular structure and only differ in the number of hydroxyl groups within the carbon6 side chain and its configuration which is $\mathrm{D}$-configurated in the case of neopterins but L-configurated in case of biopterins. However, it is unlikely that $\mathrm{D}$ and $\mathrm{L}$ configuration is of any influence in our experiments, since the dihydroforms of D-neopterin and L-biopterin are indifferent regarding the effects on lipid biosynthesis.

Biopterin is found in normal liver and other tissue $(14,15)$, and elevated biopterin in biological fluids is observed in genetically (phenylketonuria) or pharmacologically (methotrexate) induced dihydropteridine reductase deficiency $(16,17,18)$. The biological activity of biopterin derivatives is usually ascribed to its reduced form, 5,6,7,8-tetrahydrobiopterin $(15,19)$, with the exception that biopterin stimulates cell mitosis (20) and growth (21) in the protozoa Crithidia fasciculata. Obviously this organism is able to utilize biopterin in its oxidized form by reducing it probably enzymatically. In our study biopterin gave identical results as its reduced forms-sepiapterin, 7,8 -dihydrobiopterin and 5,6,7,8-tetrahydrobiopterin - in previous investigations (3). This finding could be explained if biopterin was reduced also upon incubation with rat liver homogenates. Yet, there is no further evidence for this speculation, and further studies will be necessary to address this question.
In conclusion, our results allow us to suggest that the oxidation state of pteridines is more im portant than the configuration of pterin-(6)-side chain in changes of fatty acid incorporation into phospholipids as well as in regulation of cholesterol content in samples.

\section{Acknowledgement}

This work was financially supported by the Austrian funds "Zur Förderung wissenschaftlicher Forschung", P 10776.

\section{References}

1. Rudzite V., Jurika E., Reibnegger G., Wachter H., Fuchs D. Influence of kynurenine, neopterin, noradrenalin and pyridoxal-5-phosphate on cholesterol and phospholipid content and phospholipid biosyn thesis in vitro. Pteridines, 1993: 4: 126-130.

2. Rudzite V., Jurika E., Fuchs D., Reibnegger G. Wachter $\mathrm{H}$. The influence of kynurenine, neopterin and pyridoxal-5-phosphate /P-5-P/ on cholesterol content and phospholipid biosynthesis in vitro. In: LTryptophan Current Prospects in Medicine and Drug Safety. Kochen W. and Steinhart H. Eds. Walter de Gruyter, Berlin-New York, 1994; 165-170.

3. Rudzite V., Jurika E., Baier-Bitterlich G., Wachter H., Fuchs D. Effect of sepiapterin, 7, 8-dihydrobiopterin 5, 6, 7,8-tetrahydrobiopterin and xanthopterin on cholesterol and phospholipid content and phospholipid biosynthesis in vitro. Pteridines, 1995; 6: 69-73.

4. Nilsson S. Synthesis and secretion of biliary phospholipids in man. Acta Chirurgica Scandinavica, 1970; Suppl. 405: 1-38.

5. Folch J., Lees M., Stanley G.H. A simple method for isolation and purification of total lipids from animal tissues. J. Biol. Chem., 1957; 226: 495-509.

6. Predtetchenski V.J., Borovskaja V.M., Margolina L.T. Handbook of Clinical and Laboratory Investigation, Medgiz, Moscow, 1950 (Russ).

7. Homolka J. Bestimmung des anorganischen Phosphors nach Urbach- Raabe (modifiziert). In: Chemische Diagnostik im Kindersalter mit Bevorzugung der Mikrometrischen Blutanalysen. VEB, Verlag Volk und Gesundheit, Berlin, 1964; 354-355.

8. Kates M. Technique of Lipidology. Mir, Moscow, 1978; 201-203 (Russ).

9. Weber E. Grundriss der biologischen Statistik fr Naturwissenschaftler, Landwirte und Mediziner. Gustav Fischer Verlag, Jena, 1957.

10. Leninger A. Biochemistry. The Molecular Basis of Cell Structure and Function. Mir, Moscow, 1974; pp. 603 (Russ).

11. Weiss G., Fuchs D., Hausen A., Reibnegger G., Werner E.R., Werner-Felmayer G., Semenitz E., Dierich M.P., Wachter H. Neopterin modulates toxicity mediated by reactive oxygen and chloride 
species. FEBS Lett. 1993; 321: 89-92.

12. Murr C., Fuchs D., Gössler W., Hausen A., Reibnegger G., Werner E.R., Werner-Felmayer G., Esterbauer $\mathrm{H}$., Wachter $\mathrm{H}$. Enhancement of hydrogen peroxide-induced luminol-dependent chemiluminescence by neopterin depends on the presence of iron chelator complexes. FEBS Lett. 1994; 338: 223-226.

13. Reibnegger G., Fuchs D., Murr C., Dierich M.P., Pfleiderer W., Wachter H. Effects of pteridines on lumiol-dependent chemiluminescence induced by chloramine-T. Free. Radical. Biol. Med. 1995; 18: 515-523.

14. Rembold H., Gyure W.L. Biochemistry of Pteridines. Angew. Int. Ed. Engl. 1972; 11: 1061-1072.

15. Metzler D.E. Biochemistry. Mir, Moscow, 1980; vol. 2: pp. 277 (Russ).

16. Kaufman S., Holzman N.A., Milstein S., Butler I.J., Krumholz A. Phenylketonuria due to deficiency of dihydropteridine reductase. N. Engl. J. Med. 1975; 293: 785-790.

17 Curtius H.C., Niederwieser A., Viscontini M., Otten
A., Schaub J., Scheibenreiter S., Schmidt A. Atypical phenylketonuria due to tetrahydrobiopterin deficiency. Diagnosis and treatment with tetrahydrobiopterin, dihydrobiopterin and sepiapterin. Clin. Chim.Acta, 1979; 93: 251-262.

18. Wachter H., Fuchs D., Hausen A., Reibnegger G., Weiss G., Werner E.R., Werner-Felmayer G. Neopterin. Biochemistry-Methods-Clinical Aplication. Walter de Gruyter, Berlin-New York, 1992.

19. Werner-Felmayer G.,Werner E.R., Fuchs D., Hausen A., Reibnegger G., Wachter H. Tetrahydrobiopterindependent formation of nitrite and nitrate in murine fibroblasts. J. Exp. Med., 1990; 172: 1599-1607.

20. Broquist H.P., Albrecht A.M, Pteridines and the nutrition of the protozoan Crithidia fasciculata. Proc. Soc. Exp. Biol. Med., 1955; 89: 178-180.

21. Patterson E.L., Broquist H.P., Albrecht A.M., von Saltza M.H., Stokstad E.L.R. A new pteridine in urine required for the growth of protozoan Crithidia fasciculata. J. Am. Chem. Soc., 1955; 77: 3167 3168 . 